DANIELA MARIA ROSA NASCIMENTO

\title{
ASPECTOS CONCORRENCIAIS DAS CLÁUSULAS DE PARIDADE NO COMÉRCIO ONLINE
}

\author{
DISSERTAÇÃO DE MESTRADO \\ Orientadora: Professora Titular Dra. Paula Andrea Forgioni
}

UNIVERSIDADE DE SÃO PAULO

FACULDADE DE DIREITO

São Paulo/SP

2020 



\title{
ASPECTOS CONCORRENCIAIS DAS CLÁUSULAS DE PARIDADE NO COMÉRCIO ONLINE
}

\begin{abstract}
Dissertação de Mestrado apresentada à Banca Examinadora do Programa de Pós-Graduação em Direito da Faculdade de Direito da Universidade de São Paulo, como exigência parcial para obtenção do título de Mestre em Direito, na área de concentração de Direito Comercial, sob a orientação da Profa. Titular Dra. Paula Andrea Forgioni.
\end{abstract}

UNIVERSIDADE DE SÃO PAULO

FACULDADE DE DIREITO

São Paulo/SP

2020 
Autorizo a reprodução e divulgação total ou parcial deste trabalho, por qualquer meio convencional ou eletrônico, para fins de estudo e pesquisa, desde que citada a fonte.

Nascimento, Daniela Maria Rosa

Aspectos concorrenciais das cláusulas de paridade no comércio online; Daniela Maria Rosa Nascimento; orientadora Paula Andrea Forgioni - São Paulo, 2020.

138

Dissertação (Mestrado - Programa de Pós-Graduação em Direito Comercial) - Faculdade de Direito, Universidade de São Paulo, 2020 .

1. Cláusulas de paridade. 2. Restrições verticais. 3. Condutas anticompetitivas. 4. Direito concorrencial. 5. Direito comercial. I. Forgioni, Paula Andrea, orient. II. Título. 


\section{ASPECTOS CONCORRENCIAIS DAS CLÁUSULAS DE PARIDADE NO COMÉRCIO ONLINE}

Dissertação de Mestrado apresentada à Banca Examinadora do Programa de Pós-Graduação em Direito da Faculdade de Direito da Universidade de São Paulo, como exigência parcial para obtenção do título de Mestre em Direito, na área de concentração de Direito Comercial, sob a orientação da Profa. Titular Dra. Paula Andrea Forgioni.

\section{BANCA EXAMINADORA}

\section{Presidente:}

Professora Titular Dra. Paula Andrea Forgioni

$1^{\circ}$ Examinador (a):

$2^{\mathbf{o}}$ Examinador (a):

$3^{\circ}$ Examinador (a): 
Autora: Daniela Maria Rosa Nascimento. Título: Aspectos concorrenciais das cláusulas de paridade no comércio online. 2020. 138 p. Mestrado - Faculdade de Direito, Universidade de São Paulo, São Paulo, 2020.

\section{RESUMO}

Nas últimas décadas, a sociedade observou o surgimento de um novo tipo de comércio, baseado em trocas comerciais que dispensam a presença física dos agentes envolvidos, feitas através da internet em portais conhecidos como "plataformas virtuais" - o comércio online. Essa nova realidade induziu a transposição de determinados arranjos contratuais utilizados nos negócios físicos para o meio virtual, sendo um deles a chamada "cláusula de paridade" (também conhecida como "nação mais favorecida" ou "consumidor mais favorecido"), que vem sendo objeto de investigação de diversas autoridades antitruste mundo afora. As cláusulas de paridade são disposições contratuais que estipulam que o agente interessado em vender e/ou promover bens e serviços em uma plataforma virtual não ofereça os mesmos bens e serviços a condições mais favoráveis em canal de vendas próprio e/ou em outras plataformas rivais. Este trabalho tem por objetivo estudar as cláusulas de paridade e os potenciais efeitos positivos e negativos que tais disposições podem causar no mercado, partindo-se tanto de estudos teóricos quanto de casos práticos, passados no Brasil e em outras jurisdições referência para o antitruste.

Palavras-chave: Antitruste; Comércio; Online; Condutas anticompetitivas; Restrições verticais; Cláusula de paridade; Cláusula MFN. 
Author: Daniela Maria Rosa Nascimento. Title: Competitive aspects of parity clauses in online commerce. 2020. 138 p. Master - Law School, University of São Paulo, São Paulo, 2020 .

\begin{abstract}
In the last decades, society has observed the emergence of a new type of trade, based on commercial exchanges that doesn't require physical presence of those involved, made through the internet in web portals known as "virtual platforms" - the online commerce. This new reality led to the transposition of certain contractual arrangements used in physical business to the virtual environment, one of them called "parity clause" (also known as "mostfavored-nation" or "most-favored-costumer"), that has been the subject of research by various antitrust authorities around the world. Parity clauses are contractual provisions that stipulate that an agent interested in selling and / or promoting goods and services on a virtual platform does not offer the same goods and services to more favorable terms on their own sales channel and / or other rival platforms. This work aims to investigate the parity clauses and the potential positive and negative effects that such provisions can have on the market, based on both theoretical and practical case studies from Brazil and other antitrust reference jurisdictions.
\end{abstract}

Keywords: Antitrust; Commerce; Online; Anticompetitive conducts; Vertical restraints; Parity clause; MFN Clause. 
1. INTRODUÇÃO ........................................................................................................10

2. O COMÉRCIO ONLINE E PLATAFORMAS VIRTUAIS.......................................13

2.1. Plataformas virtuais: caracterização .............................................................................13

2.2. Mercados de dois lados e efeitos de rede .................................................................15

2.3. Eficiências associadas ao comércio online .................................................................... 18

2.3.1. Redução dos custos de pesquisa e assimetria informacional do consumidor

2.3.2. Expansão do alcance do comércio global ..........................................................20

2.3.3. Visibilidade para agentes econômicos de pequeno e médio porte..................21

3. CLÁUSULA DE PARIDADE: CARACTERIZAÇÃO ..............................................22

3.1. Conceituação ...................................................................................................................22

3.1.1. Cláusula de paridade como tipo de cláusula da nação mais favorecida......22

3.1.2. A cláusula de paridade como tipo de acordo de relação de preços (Price Relationship Agreements) .......................................................................................................27

3.2. A cláusula de paridade e o comércio online ..............................................................29

3.3. A cláusula de paridade como espécie de restrição vertical......................................30

4. EFICIÊNCIAS ASSOCIADAS ÀS CLÁUSULAS DE PARIDADE ............................33

4.1. Redução dos custos de transação .................................................................................33

4.2. Prevenção do "efeito carona" (free-riding effect)......................................................35

4.3. Redução do problema do showrooming ......................................................................36

4.4. Proteção da marca ..............................................................................................38

5. POTENCIAIS EFEITOS ANTICOMPETITIVOS DAS CLÁUSULAS DE

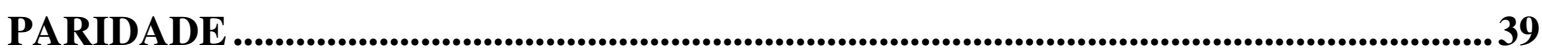

5.1. Intermediação excessiva.....................................................................................................40

5.2. Arrefecimento da competição via preços.......................................................................43

5.2.1. O caso dos Price Comparison Websites (PCW)....................................................45

5.3. Barreiras à entrada e aumento dos custos de rivais ..................................................48

5.4. Redução dos incentivos ao investimento e à inovação..............................................55

5.5. $\mathrm{O}$ caso Amazon ............................................................................................................56

5.6. O caso das agências de viagem online - Online Travel Agencies (OTA) ..............59

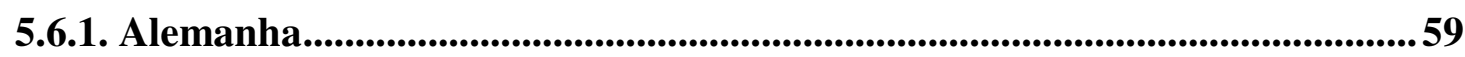

5.6.1.1. Bundeskartellamt e HRS................................................................................59

5.6.1.2. Bundeskartellamt e Booking.com.....................................................................63

5.6.2. França .....................................................................................................................................66 
5.6.3. Itália 67

5.6.4. Estados Unidos da América ........................................................................68

5.6.5. A dinâmica de precificação de hotéis após decisões dos casos OTA ............. 70

5.6.6. Considerações gerais sobre o caso das OTA ................................................77

5.7. Facilitação da formação de cartéis.....................................................................79

5.7.1. Os cartéis hub-and-spoke .............................................................................82

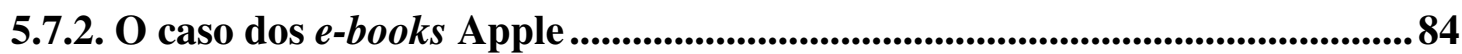

5.7.2.1. Caso Apple na Comissão Europeia ..............................................................90

5.7.2.2. Considerações gerais do caso Apple ...........................................................92

5.8. Considerações gerais sobre os possíveis efeitos anticompetitivos das cláusulas de paridade ............................................................................................................................93

6. CLÁUSULAS DE PARIDAdE NO CONTEXTO BRASILEIRO ..............................95

6.1. A legislação brasileira de defesa da concorrência ................................................95

6.1.1. Breve considerações sobre padrões probatórios............................................97

6.1.2. As cláusulas de paridade na Lei n. ${ }^{0} 12.529 / 2011$............................................ 102

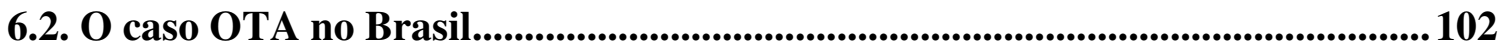

6.2.1. A Representação do FOHB.................................................................................. 104

6.2.2. As defesas das representadas................................................................................. 106

6.2.3. Os Termos de Compromisso de Cessação de Prática e desfecho do caso.. 107

6.3. Horizontes para o tratamento das cláusulas de paridade no Brasil ................... 109

7. SÍNTESE CONCLUSIVA ............................................................................................... 118

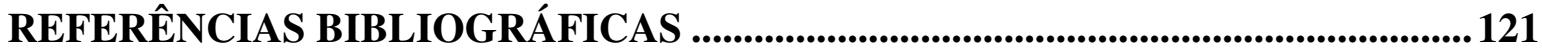

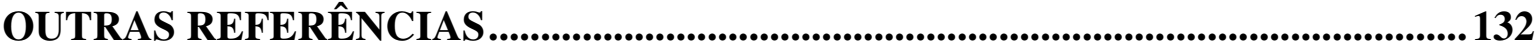




\section{INTRODUÇÃO}

A expansão da internet vem deslocando o ambiente de transações comerciais do meio físico para o meio virtual. No Brasil, em 2018, o comércio eletrônico cresceu cerca de $12 \%^{1}$, e estimativas mais recentes apontam para um aumento de $16 \%$ no volume de transações em 2019. ${ }^{2}$ A conveniência do comércio online aliada à confiabilidade, cada vez maior, depositada pelos consumidores nas empresas que atuam nesse segmento tem impulsionado essa transição, que rapidamente vem alterando a maneira de se fazer negócios no mundo.

Uma crescente expansão no meio eletrônico é o modelo de negócio das plataformas virtuais, atuais protagonistas do comércio online. Por meio dessas plataformas, os consumidores podem ter acesso a inúmeros bens e serviços em um único ambiente, sem necessidade de qualquer deslocamento físico. Esses ambientes virtuais são usualmente comparados com shopping centers, onde vendedores e compradores encontram-se para realizar transações comerciais. ${ }^{3}$

O crescimento das plataformas virtuais e do comércio eletrônico como um todo promoveu a transposição de determinados arranjos contratuais, outrora utilizados nos negócios do meio físico, também para o meio virtual. Um desses arranjos é a cláusula de paridade (usualmente chamada de "nação mais favorecida" ou "consumidor mais favorecido" $)^{4}$, que vem sendo objeto de investigação de diversas autoridades antitruste mundo afora.

Em linhas gerais, as cláusulas de paridade são disposições contratuais que determinam que o agente interessado em promover bens e serviços em uma plataforma virtual

\footnotetext{
${ }^{1}$ Dados extraídos de: <https://www.ecommercebrasil.com.br/noticias/e-commerce-fatura-2018-ebit-nielsen/> Acesso em: 15/08/2019.

2 Dados extraídos de: <https://www.ecommercebrasil.com.br/noticias/comercio-eletronico-deve-crescer-16no-pais-em-2019-preve-abcomm/> Acesso em: 15/08/2019.

${ }^{3}$ AKMAN, Pinar. A Competition Law Assessment of Platform Most-Favoured-Customer Clauses. Centre For Competition Policy, 2015, p. $1 . \quad$ Disponível $<$ https://papers.ssrn.com/sol3/papers.cfm?abstract_id=2669395 > . Acesso em: 08/09/2018.

${ }^{4}$ Por exemplo, em: BOIK, Andre; CORTS, Kenneth S. The Effects of Platform MFNs on Competition and Entry. University of Toronto, junho, 2014. Disponível em: <https://editorialexpress.com/cgibin/conference/download.cgi?db_name=RES2015\&paper_id=41>. Acesso em: 10/05/2018
} 
não ofereça os mesmos bens e serviços a condições mais favoráveis em canal de vendas próprio e/ou em outras plataformas rivais. ${ }^{5}$

Quando plataformas virtuais adotam cláusulas de paridade em seus contratos, visam a garantir que o vendedor não se utilize de seu ambiente para mera divulgação de seus produtos e/ou serviços, oferecendo-os a preços menores em seu próprio canal de vendas ou em plataformas rivais que cobram taxas mais reduzidas. Assim, tal previsão contratual garante que a plataforma por ela beneficiada terá o melhor preço disponível para aquele bem ou serviço, impedindo que o próprio vendedor ou terceiros tirem proveito de seu investimento.

Do ponto de vista da análise concorrencial, há relativo consenso de que as cláusulas de paridade podem gerar tanto efeitos pró-competitivos quanto efeitos anticompetitivos $^{6}$, de forma que não são, atualmente, consideradas per se ilícitas.

Todavia, algumas características próprias das plataformas virtuais, notadamente os efeitos de rede indiretos, tendem a formar mercados concentrados, potencializando os possíveis efeitos anticompetitivos dessas cláusulas, tais como (i) uniformização de preços; (ii) criação de barreiras à entrada de novas plataformas com menores custos; (iii) exclusão de plataformas rivais; e (iv) facilitação da formação de cenários colusivos. ${ }^{7}$

Jean Tirole, economista laureado com o prêmio Nobel, em entrevista de 2017 à Econ Focus, abordou essa relação entre a tendência de formação de mercados concentrados no ambiente online, em razão da atuação de plataformas virtuais, e como as cláusulas de paridade podem reforçar essa concentração. Ao responder se as plataformas virtuais levantariam questões especiais à regulação antitruste, Tirole afirma que:

Eu acredito que a resposta é sim - parcialmente porque as novas plataformas possuem tendência natural ao monopólio, ao exibirem relevantes externalidades de rede. (...) Externalidades de rede tendem a criar monopólios ou pequenos oligopólios. (...) Outra questão é que a maior parte das plataformas oferecem

\footnotetext{
${ }^{5}$ EZRACHI, Ariel. The Competitive Effects of Parity Clauses on Online Commerce. Oxford Legal Studies Research Paper No. 55/2015, p. $1 . \quad$ Disponível em: $<$ https://papers.ssrn.com/sol3/papers.cfm?abstract_id=2672541> Acesso em: 08/09/2018.

${ }^{6}$ GÜRKAYNAK, Gönenç; GÜNER, Ayse; DINIZ, Sinan; FILSON, Janelle. Most-favored-nation clauses in commercial contracts: legal and economic analysis and proposal for a guideline. In: European Journal of Law and Economics, 42:129-155, 2016, p. $1 . \quad$ Disponível em: $<$ https://papers.ssrn.com/sol3/papers.cfm?abstract_id=3150371>. Acesso em: 18/09/2018.

${ }^{7}$ BAKER, Jonathan B; SCOTT MORTON, Fiona. Antitrust Enforcement Against Platform MFNs. In: The Yale Law Journal, Vol. 127, no 7, 2018, p. 2181. Disponível em: <https://www.yalelawjournal.org/feature/antitrustenforcement-against-platform-mfns >. Acesso em: 18/09/2018.
} 
garantias de melhor preço, também chamadas cláusulas da "nação mais favorecida" ou cláusula de paridade de preço. Você, como consumidor, tem a garantia de que o preço ofertado na plataforma será o menor existente, conforme exigido dos vendedores. Parece bom, exceto que se todos ou a maior parte dos vendedores estão listados na plataforma, e a plataforma garante o menor preço, não há nenhum incentivo para você olhar em outro lugar - você se tornou um consumidor "único", e então a plataforma pode cobrar taxas altas para que o vendedor tenha acesso a você. (...) Isso pode algumas vezes ser bem problemático para a concorrência. ${ }^{8}$

Este trabalho tem o objetivo de analisar as características das cláusulas de paridade e os efeitos por elas produzidos no mercado, tanto benéficos quanto prejudiciais. Serão estudados os principais casos investigados até o momento na Europa, Estados Unidos e Brasil, com o propósito de estabelecer um panorama do estado da arte atual sobre o tema e o que se pode esperar nos próximos anos em termos de política antitruste direcionada a essas cláusulas.

Para tanto, estrutura-se este trabalho da seguinte forma: (i) a primeira parte descreve o comércio eletrônico e suas principais características, com especial foco nas plataformas virtuais; (ii) a segunda parte é dedicada à conceituação das cláusulas de paridade, abarcando as principais definições encontradas na literatura especializada; (iii) a terceira parte trata das eficiências mais importantes associadas às cláusulas de paridade; (iv) a quarta parte analisa os efeitos anticompetitivos mais comumente associados a tais cláusulas e as principais teorias do dano apontadas pela literatura, mediante o exame de casos concretos enfrentados por jurisdições estrangeiras; (v) a quinta parte trata do estado atual do tema no Brasil, tanto em termos legislativos quanto de precedentes analisados pelo Conselho Administrativo de Defesa Econômica (CADE), e reflete sobre os horizontes do tratamento das cláusulas de paridade no Brasil a partir das ponderações apresentadas; (vi) e, por fim, encerra-se com uma síntese conclusiva dos assuntos tratados ao longo da exposição.

\footnotetext{
${ }^{8}$ TIROLE, Jean. [Entrevista concedida a] David A. Price. In: Econ Focus Magazine, Fourth Quarter 2017. Disponível em: <https://www.richmondfed.org/publications/research/econ_focus/2017/q4/interview> Acesso em: 27/09/2019. Tradução livre.
} 


\section{SÍNTESE CONCLUSIVA}

Neste trabalho, buscou-se traçar as características das cláusulas de paridade e identificar os potenciais efeitos que poderiam produzir no mercado, tanto positivos, do ponto de vista das eficiências, quanto negativos, consubstanciados por eventuais impactos negativos na concorrência. Para tanto, recorreu-se à literatura especializada, tanto nacional quanto estrangeira, e ao estudo de casos recentes enfrentados por diversas autoridades antitruste em jurisdições com longa tradição no antitruste.

Em suma, os resultados obtidos indicam que, em geral, há justificativa econômica e negocial para a existência de cláusulas de paridade em contratos de plataformas de comércio online, relacionadas essencialmente à proteção do investimento por meio da coibição de comportamentos oportunistas, tanto pelos próprios consumidores (showrooming) quanto pelos demais players do mercado (free-riding), representados por vendedores/anunciantes e outras plataformas, respectivamente.

Entende-se que a cláusula de paridade passou a ser utilizada como ferramenta pelas plataformas virtuais para garantir o acesso e compra efetiva pelos consumidores através de seus portais, pois impede a oferta de preços menores em quaisquer outros canais de venda (cláusula ampla) ou, ao menos, em canal de vendas próprio do vendedor (cláusula restrita).

A importância da cláusula de paridade é ainda reforçada pela externalidade de rede inerente às plataformas virtuais de dois (ou mais) lados. A existência da massa crítica de usuários, i.e., quantidade suficiente para garantir a viabilidade da plataforma, depende do atingimento de um número de usuários relevante em todos os lados, consubstanciando o que se convencionou chamar de "efeitos de rede indiretos".

Assim, por conta dos efeitos de rede indiretos, muitas plataformas virtuais buscam ferramentas aptas a garantir a manutenção da massa crítica de usuários. Essas ferramentas, porém, podem gerar prejuízos efetivos à concorrência, dificultando o desenvolvimento de novos negócios e/ou a sustentação de um ambiente competitivo saudável.

No caso das cláusulas de paridade, por exemplo, foram identificados alguns possíveis efeitos negativos na concorrência: (i) intermediação excessiva por parte das plataformas virtuais; (ii) arrefecimento da competição via preços; (iii) aumento das barreiras à entrada de novos agentes; (iv) redução dos incentivos ao investimento e inovação; e (v) 
facilitação da formação de cartéis. Esses efeitos foram explorados por diversas agências antitruste nos casos-paradigma dos Price Comparison Websites, caso Amazon, caso das Online Travel Agencies e caso Apple.

Dentro do contexto brasileiro, buscou-se analisar o enquadramento legal das cláusulas de paridade no âmbito da Lei n. ${ }^{\circ}$ 12.529/2011, bem como traçar os padrões probatórios usualmente aplicados pela autoridade antitruste brasileira e delinear o que seria mais adequado no que se refere ao tratamento do tipo de cláusula aqui retratado.

Analisando-se o cenário brasileiro, é possível verificar que o tema das cláusulas de paridade foi objeto de estudos de diversos acadêmicos, ainda que a casuística seja bastante reduzida. A bem da verdade, há apenas um caso enfrentado pelo CADE cujo objeto principal era a investigação dos possíveis efeitos anticompetitivos das cláusulas de paridade - o chamado caso das Online Travel Agencies, que permanece como referência de estudo desse tipo de conduta.

A ausência de mais casos sobre o tema, entretanto, não pode ser lida como a negativa de existência de potenciais efeitos deletérios à concorrência a partir da utilização de cláusulas de paridade. Com efeito, o CADE tanto reconheceu essa potencialidade como foram celebrados acordos com as representadas, cujo objetivo foi mitigar os prejuízos identificados pela autoridade. Ademais, não raro contratos padrão celebrados por plataformas de marketplace com vendedores/anunciantes contêm cláusulas de paridade, tanto restritas quanto amplas, não obstante o posicionamento do CADE desfavorável à forma ampla da disposição, o que pode vir a gerar questionamentos futuros de agentes econômicos prejudicados (ou até mesmo de consumidores).

De toda forma, o tratamento das cláusulas de paridade pelas autoridades de defesa da concorrência não foge ao padrão inerente a qualquer intervenção do Estado no funcionamento do mercado - antes de mais nada, é de suma importância que a intervenção seja suficiente para coibir o abuso, mas não excessiva a ponto de frustrar os incentivos à inovação dos agentes econômicos.

É preciso sopesar, a todo momento, qual medida é adequada aos objetivos pretendidos - se a intervenção, visando a sinalizar aos agentes econômicos que determinadas condutas não serão toleradas, por consubstanciarem alguma forma de abuso, ou a abstenção do escrutínio estatal, a fim de evitar exageros regulatórios que poderiam prejudicar o mercado ao dificultar a inovação e o surgimento de novos modelos de negócio. 
De toda forma, a questão não é nova e sem dúvidas continuará a permear o debate sempre que se estiver diante de uma conduta possivelmente anticompetitiva, mas que possa gerar eficiências economicamente aproveitáveis. 


\section{REFERÊNCIAS BIBLIOGRÁFICAS}

AGHION, P., \& BOLTON, P. Contracts as a barrier to entry. In: The American Economic Review, vol. 77(3), 1987, pp. 388-401. Disponível em: < https://www.jstor.org/stable/1804102> Acesso em: 25.07.2019.

AKMAN, Pinar. A Competition Law Assessment of Platform Most-Favoured-Customer Clauses. In: Centre For Competition Policy, 2015. Disponível em: $<$ https://papers.ssrn.com/sol3/papers.cfm?abstract_id=2669395>. Acesso em: 08/09/2018.

SOKOL, Daniel. Online RPM and MFN Under Antitrust Law and Economics. In: Review of Industrial Organization, Vol. 50, 2017. Disponível em: $<$ https://link.springer.com/article/10.1007/s11151-016-9560-x $>$. Acesso em: 10/05/2018.

AMORIM, Fernando. A ilicitude pelo objeto e o alcance da discricionariedade do CADE no processo administrativo sancionador antitruste. In: Revista de Defesa da Concorrência, Vol. $5, \mathrm{n}^{\mathrm{o}} 2,2017$.

AREEDA, Phillip E.; HOVENKAMP, Herbert. Antitrust Law: an analysis of antitrust principles and their application. Vol. VII. 2.ed. Wolters Kluwers, 2003.

KAPLOW, Louis. Antitrust analysis: problems, texts, cases. $7^{\text {a }}$ Ed., Aspen Casebook, Wolters Kluwer, 2013.

ARMSTRONG, Mark. Competition in Two-Sided Markets. In: RAND Journal of Economics, 2006, pp. 669-670.

ATHAYDE, Amanda. Antitruste, varejo e infrações à ordem econômica. São Paulo: Singular, 2017.

BAKER, Jonathan B. Market Concentration in the Antitrust Analysis of Horizontal Mergers. In: Antitrust Law and Economics, 2010.

Vertical Restraints with Horizontal Consequences: Competitive Effects of “Most-Favoured-Customer” Clauses. In: Antitrust Law Journal, Vol. 64, No. 3, 1996, pp. 517-534. Disponível em: <https://www.jstor.org/stable/40843338> Acesso em: 05/09/2019. CHEVALIER, Judith A. The Competitive Consequences of MostFavored-Nation Provisions. In: Law Reviews and Other Academic Journals, Paper 227, 
2013. Disponível em: <https://digitalcommons.wcl.american.edu/facsch_lawrev/277> Acesso em: 10/09/2018.

SCOTT MORTON, Fiona. Antitrust Enforcement Against Platform MFNs. In: The Yale Law Journal, Vol. 127, $\mathrm{n}^{\mathrm{o}}$ 7, 2018. Disponível em: $<$ https://www.yalelawjournal.org/feature/antitrust-enforcement-against-platform-mfns $>$. Acesso em: 18/09/2018.

BLOOMBERG. Expedia Under Investigation by Utah Over Hotel Collusion Claims. In: Bloomberg, 09/05/2019. Disponível em: <https://www.bloomberg.com/news/articles/201905-09/expedia-under-investigation-by-utah-over-hotel-collusion-claims > Acesso em: 05/09/2019.

BOIK, Andre. CORTS, Kenneth S. The Effects of Platform MFNs on Competition and Entry. University of Toronto, junho, 2014. Disponível em: <https://editorialexpress.com/cgibin/conference/download.cgi?db_name=RES2015\&paper_id=41 $\geq$. Acesso em: 10/05/2018.

BORK, Robert. The Antitrust Paradox. New York: Basic Books, 1978.

BRUNA, Sérgio Varella. O poder econômico e a conceituação do abuso em seu exercício. 1a Ed. São Paulo: Editora Revista dos Tribunais, 2001.

BUCCIROSSI, Paulo. Background Note - OECD Policy Roundtables, Vertical Restraints for Online 2013. Sales, Disponível em: < http://www.oecd.org/competition/VerticalRestraintsForOnlineSales2013.pdf> Acesso em: 20/09/2019.

Parity Clauses: Economic incentives, theories of harm and efficiency justifications. In: Competition Law \& Policy Debate, 1(3), 2015, p. 43-52.

BUNDESKARTELLAMT. Narrow 'best price' clauses of Booking also anticompetitive. In: Bundeskartellamt, $23 / 12 / 2015$. Disponível em:<https://www.Bundeskartellamt.de/SharedDocs/Meldung/EN/Pressemitteilungen/2015/ 23_12_2015_Booking.com.html> Acesso em: 07/08/2019.

BUSINESS INSIDER. Amazon in ending its restrictive pricing practice. In: Business Insider, 13/03/2019. Disponível em: <https://www.businessinsider.com/amazon-ends-restrictivepricing-parity-2019-3> Acesso em: 05/09/2019. 
BUTZ, David. Durable-good monopoly and best-price provisions. In: The American Economic Review, 80(5), 1062-1076, 1988. Disponível em: $<$ https://www.jstor.org/stable/2006761>. Acesso em: 10/09/2018.

CADE. CADE e associação norte americana de advogados promovem seminário sobre concorrência. In: CADE, 10/07/2018. Disponível em: $<$ http://www.cade.gov.br/noticias/cade-e-associacao-norte-americana-de-advogadospromovem-seminario-sobre-concorrencia> Acesso em: 20/08/2019

CAILlAUD, Bernard. JULLIEN, Bruno. Chicken and Egg: Competition among Intermediation Service Providers. In: RAND Journal of Economics, 34, 2003.

CALZADA, Joan, MANNA, Ester, MANTOVANI, Andrea. Platform Price Parity Clauses and Segmentation. In: UB Economics Working Papers, Collecció d'Economia E19/387. Disponível em:< https://ssrn.com/abstract=3329157> Acesso em: 07/08/2019.

CHEN, Jihui. LIU, Qihong. The Effect of Most-Favored-Customer Clauses on Prices. In: The Journal of Industrial Economics, Vol. 59, No. 3 (September 2011), pp. 343-371. Disponivel em: <https://www.jstor.org/stable/41289458>. Acesso em: 20/09/2018.

CLARK, Travis. Swiping Through the Pages of Apple's E-Book Saga: A Comment on HubAnd-Spoke Conspiracies Under the Sherman Act. Seton Hall Law, Law School Student Scholarship, 2017.

COASE, Ronald H. Durability and Monopoly. In: Journal of Law and Economics, Vol. 15, No. 1, pp. 143-149. Disponível em: <http://www.jstor.org/stable/725018>. Acesso em: $10 / 09 / 2018$. The firm, the Market and the law. University of Chicago Press, 1988.

COLANGELO, Margherita. Parity Clauses and Competition Law in Digital Marketplaces: The Case of Online Hotel Booking. In: Journal of Competition Law and Practice, 3-14, 2017, p. 15. Disponível em: < https://papers.ssrn.com/sol3/papers.cfm?abstract_id=2963503> Acesso em: 27/07/2019.

COOPER, Thomas E. Most-favored-customer Pricing and Tacit Collusion. In: The RAND Journal of Economics, Vol. 17, No. 3, Autumn, 1986, pp. 377-388. 
CORDOVIL, Leonor; CARVALHO, Vinícius Marques de; BAGNOLI, Vicente; ANDERS, Eduardo Caminati. Nova Lei de Defesa da Concorrência Comentada. São Paulo: Editora Revista dos Tribunais, 2011.

CRETELLA NETO, José. Teoria Geral das Organizações Internacionais, $3^{\text {a }}$ Ed. São Paulo: Saraiva, 2013.

CROCKER, K. LYON, T. What do 'facilitating practices' facilitate? Na empirical investigation of most-favored-nation clauses in natural gas contracts. In: Journal of Law \& Economics, 37(2), pp. 297-322. Disponível em: <https://www.jstor.org/stable/725734>.

D'ASPREMONT, Claude; FERREIRA, Rodolphe dos Santos. Meet-or-Release and MostFavored-Customer Clauses with Price-Quantity Competition Yield Cournot Outcomes. Économie publique, 17, 2005.

DENNIS, Anthony J. Potential Anticompetitive Effects of Most Favored Nation Contract Clauses in Managed Care and Health Insurance Contracts. In: Annals of Health Law, 4(1), 1995, pp.71-85. Disponível em: <https://pdfs.semanticscholar.org/5c90/484a9fc21acce8d7aec3defda434cf8eb61b.pdf> Acesso em: 03/11/2018.

EBOOK FRIENDLY. A timeline of Kindle devides and services. In: Ebook Friendly, 14/07/2019. Disponível em: <https://ebookfriendly.com/timeline-kindle-history/> Acesso em: 03/08/2019.

EISENBERG, Jonathan M.; Miller, Adam; BURROUGHS, Amelia F. California Antitrust and Unfair Competition Law - Chapter 10: Antitrust and the Internet, updated January 2016.

EVANS, David S. Competition and Regulatory Policy for Multi-sided Platforms with Applications to the Web Economy. In: Concurrences, No. 2, pp. 57-62, 2008. Disponível em: $<$ https://papers.ssrn.com/sol3/papers.cfm?abstract_id=1090368> Acesso em: 29/09/2018.

SCHMALENSEE, Richard, The Antitrust Analysis of Multi-Sided Platform Businesses. Roger Blair and Daniel Sokol, eds., Oxford Handbook on International Antitrust Economics, Oxford University Press, Forthcoming; University of Chicago Institute for Law \& Economics Olin Research Paper No. 623, 2013. Disponível em: $<$ https://ssrn.com/abstract=2185373 > Acesso em: 10/10/2018. 
EZRACHI, Ariel, The Competitive Effects of Parity Clauses on Online Commerce. Oxford Legal Studies Research Paper No. 55/2015. Disponível em: $<$ https://papers.ssrn.com/sol3/papers.cfm?abstract_id=2672541>. Acesso em: 08/09/2018.

EU Competition Law: An Analytical Guide to the Leading Cases. Third Edition. Oxford and Portland, Oregon, 2016.

FEDERICO, Giulio. SCOTT MORTON, Fiona M. SHAPIRO, Carl. Antitrust and Innovation: Welcoming and Protecting Disruption. In: NBER, Innovation Policy and the Economy, 2019. Disponível em: <http://faculty.haas.berkeley.edu/shapiro/disruption.pdf> Acesso em: 20/09/2019.

FLETCHER, Amelia. HVIID, Morten. Broad Retail Price MFN Clauses: Are They RPM “At Its Worst”? In: Antitrust Law Journal, Vol. 81, 2016, p. 65-98. Disponível em: $<$ https://ueaeprints.uea.ac.uk/60189/>. Acesso em: 18/09/2018.

FORGIONI, Paula A. Contrato de Distribuição. São Paulo: Editora Revista dos Tribunais, 2005.

Direito Concorrencial e Restrições Verticais. São Paulo: Editora Revista dos Tribunais, 2007.

Os Fundamentos do Antitruste. $8^{\mathrm{a}}$ Ed. São Paulo: Editora Revista dos

Tribunais, 2015.

FRIEDERISZICK, Hans W. GLOWICKA, Ela. Competition policy in modern retail markets. In: Journal of Antitrust Enforcement, 2016.

GERADIN, Damien; PEREIRA NETO, Cario Mario da Silva. Restrições verticais adotadas por empresas dominantes: uma análise do Direito Concorrencial no Brasil e na União Europeia. Vol. 2, São Paulo: Editora Revista dos Tribunais, 2013.

GONÇALVES, Priscila Brólio. Fixação e sugestão de preços de revenda em contratos de distribuição: análise dos aspectos concorrenciais. São Paulo: Singular, 2002.

GONZÁLEZ-DIAZ, Francisco Enrique. BENNETT, Matthew. The law and economics of most-favoured nation clauses. In: Competition Law \& Policy Debate. Vol. 1, Issue 3, agosto, 2015. Disponível

em: 
$<$ https://awards.concurrences.com/IMG/pdf/the_law_and_economics_of_mostfavoured_nation_clauses.pdf>. Acesso em: 10/09/2018.

GÜRKAYNAK, Gönenç. GÜNER, Ayse. DINIZ, Sinan. FILSON, Janelle. Most-favorednation clauses in commercial contracts: legal and economic analysis and proposal for a guideline. In: European Journal of Law and Economics, 42:129-155, 2016. Disponível em: $<$ https://papers.ssrn.com/sol3/papers.cfm?abstract_id=3150371>. Acesso em: 18/09/2018.

HAUCAP, Justus. HEIMESHOFF, Ulrich. Google, Facebook, Amazon, eBay: Is the Internet Driving Competition or Market Monopolization? In: Discussion paper, No. 83, Düsseldorf Institute for Competition Economics, janeiro, 2013.

HILDEBRAND, Doris. Economic Analyses of vertical agreements - A self-assessment. In: International Competition Law Series, Kluwer Law International, 2005.

HINCHLIFFE, Susan. Price Relationship Agreements: Procompetitive, Anticompetitive or Neutral? In: American Bar Association, Antitrust, Spring, 2013.

HORTACSU, Ali MARTINEZ-JEREZ, Francisco de Asis. DOUGLAS, Jason, The Geography of Trade on Ebay and Mercadolibre. In: NET Institute Working Paper No. 0609, 2006. Disponível em: <https://ssrn.com/abstract=939327>. Acesso em: 24/10/2018.

HOTEL MANAGEMENT. Now Austria refuses "to play ball” on rate parity. In: Hotel Management, 20/12/2016. Disponível em: <https://www.hotelmanagement.net/salesmarketing/now-austria-refuses-to-play-ball-rate-parity> Acesso em: 19/09/2019.

HOVENKAMP, Erik. Antitrust Policy for Two-Sided Markets (February 9, 2018). In: Journal of Corporation Law (2018 Forthcoming).

Platform Antitrust. In: Journal of Corporation Law, 2019.

HOVENKAMP, Herbert. Federal Antitrust Policy: The Law of Competition and It's Practice. In: Hornbook Series, Fourth Edition, 2011.

HOVENKAMP, Herbert J. The Antitrust Standard for Unlawful Exclusionary Conduct. Faculty Scholarship, 1777, 2008. 
HUNOLD, Matthias. KESLER, Reinhold. LAITENBERGER, Ulrich. SCHLUTTER, Frank. Evaluation of Best Price Clauses in Online Hotel Bookings. In: International Journal of Industrial Organization, Vol. 61, 2018, pp. 542-571.

HVIID, Morten. Vertical Agreements Between Suppliers and Retailers that Specify a Relative Price Relationship Between Competing Products or Competing Retailers. OECD, Hearing on Across Platform Parity Agreements. Outubro, 2015. Disponível em: $<$ http://www.oecd.org/officialdocuments/publicdisplaydocumentpdf/?cote=DAF/COMP(20 15)6\&doclanguage=en $\geq$ Acesso em: 30/09/2018.

JOHANSEN, Bjørn Olav. VERGÉ, Thibaud. Platform price parity clauses with direct sales. University of Bergen, Department of Economics, 2017. Disponível em: <https://pdfs.semanticscholar.org/9f66/ef3ee90b113eaf8515e7e2c1cbb9858a2146.pdf> Acesso em: 21/08/2019.

JOHNSON, Justin P. The Agency and Wholesale Models in Electronic Content Markets. 2018. Disponível em: < https://pdfs.semanticscholar.org/4de8/8c05120fd7f23a4b5419098dce2c47220d3d.pdf> Acesso em: 08/05/2019.

The Agency Model and MFN Clauses. Cornell University, 2014. Disponível em: <https://papers.ssrn.com/sol3/papers.cfm?abstract_id=2217849>. Acesso em: 08/05/2018.

KHAN, Lina M. Amazon's Antitrust Paradox. Yale Law Journal, 126:710, 2017.

KLEN, Benjamin. The Apple E-Books Case: When is a Vertical Contract a Hub-and-Spoke Conspiracy? In: Journal of Competition Law and Economics, 2017. Disponível em: < https://papers.ssrn.com/sol3/papers.cfm?abstract_id=3018841 > Acesso em: 03/07/2019.

LÁFER, Celso. O GATT, a cláusula da nação mais favorecida e a América Latina. In: Revista de Direito Mercantil, Industrial, Econômico e Financeiro, v. 10, n. 3, 1971.

LEAR. Can 'Fair' Prices Be Unfair? A Review of Price Relationship Agreements. A report prepared for the Office of Fair Trading, OFT1438, 2012. Disponível em: $<$ http://www.learlab.com/wp-content/uploads/2016/04/Can\%E2\%80\%98Fair\%E2\%80\%99-Prices-Be-Unfair_-A-Review-of-Price-RelationshipAgreements.pdf $\geq$ Acesso em: 30.09.2018. 
LIANOS, Ioannis. Collusion in Vertical Relations Under Article 81 EC. Common Market Law Review, Vol. 45, No. 4, 2008. Disponível em: <https://ssrn.com/abstract=1089681> Acesso em: 03/11/2018.

MANDORFF, Martin. SAHL, Johan. The Role of the 'Equally Efficient Competitor' in the ASSESSMENT OF Abuse OF Dominance. In: Konkurrensverket Working Papers in Law and Economics, 2013. Disponível

em: $<$ http://www.konkurrensverket.se/globalassets/english/publications-and-decisions/the-roleof-the-equally-efficient-competitor-in-the-assessment-of-abuse-of-dominance.pdf $>$ Acesso em: 20/09/2019.

MANSOUR, Amine. Identifying the exclusionary effect of across-platform parity agreements: testing the tests. In: The Antitrust Bulletin, April 2018. Disponível em: $<$ https://journals.sagepub.com/doi/abs/10.1177/0003603X18770065> Acesso em: 23/07/2019.

MANTOVANI, Andrea. PIGA, Claudio. REGGIANI, Carlo. Much ado about nothing? Online platform price parity clauses and the EU Booking.com case. In: SSRN International Journal, $\quad 2019 . \quad$ maio, Disponível em:< https://papers.ssrn.com/sol3/papers.cfm?abstract_id=3381299> Acesso em: 27/07/2019.

PIGA, Claudio. REGGIANI, Carlo. On the Economic Effects of Price Parity Clauses - What Do We Know Three Years Later?. In: Journal of European Competition Law \& Practice, Volume 9, Issue 10, December 2018, pp. 650-654.

MARUYAMA, Masayoshi, ZENNYO, Yusuke. Platform Most-Favored-Customer Clauses and Investment Incentives. Kobe University, 2018.

MELLO, Gabriela. Comércio eletrônico brasileiro deve crescer 12\% em 2018. In: Exame, abril, 09/03/2018. < <ttps://exame.abril.com.br/economia/comercio-eletronico-brasileirodeve-crescer-12-em-2018/> Acesso em: 10/09/2018.

MOTTA, Massimo. Competition Policy: Theory and Practice. Cambridge University Press, 2004.

ODUDU, Okeoghene. Hub and Spoke Collusion. In: Handbook on European Competition Law, 2013, pp. 242-258. 
OECD. Most-Favoured-Nation Treatment in International Investment Law. In: OECD, 2004. Disponível em: <https://www.oecd.org/daf/inv/investment-policy/WP-2004_2.pdf>. Acesso em: 29/09/2018.

OECD. Vertical Restraints for On-line Sales. In: OECD, 2013, p. 6. Disponível em: $<$ http://www.oecd.org/competition/VerticalRestraintsForOnlineSales2013.pdf $>$. Acesso em: 08/09/2018.

OLIVEIRA, Marcelo Nunes de. Concorrência.com: cláusulas de paridade, MFNs e agências de turismo on-line. In: JOTA, 02/04/2018. Disponível em: <https://www.jota.info/opiniaoe-analise/artigos/concorrencia-com-clausulas-de-paridade-mfns-e-agencias-de-turismo-online-02042018> Acesso em: 20/08/2019.

ORBACH, Barak. Hub-and-Spoke Conspiracies. In: Arizona Legal Studies Discussion Paper No. 16-11, 15 Antitrust Source, No. 3, 2016. Disponível em: $<$ https://ssrn.com/abstract=2765476 > Acesso em: 03/11/2018.

OXERA, Parity Agreements and theories of harm in the hotel booking sector: a "no-show"? Agenda, junho, 2017. Disponível em: <https://www.oxera.com/agenda/parity-agreementsand-theories-of-harm-in-the-hotel-booking-sector-a-no-show/> Acesso em: 08/05/2018.

PETIT, Nicolas. HENRY, David. Vertical Restraints under EU Competition Law: Conceptual Foundations and Practical Framework. 2010. Disponível em: $<$ https://ssrn.com/abstract=1724891>. Acesso em: 24/10/2018.

POSNER, Richard A. Antitrust Law: An Economic Perspective, Chicago: University of Chicago Press, 1976.

The Chicago School of Antitrust Analysis, 127 U. Pa. L. Rev., 925, 932 (1979).

ROCHET, Jean-Charles. TIROLE, Jean. Platform Competition in Two-Sided Markets. In: Journal of European Economic Association, 2003.

TIROLE, Jean. Two-Sided Markets: A Progress Report. In: The RAND Journal of Economics. Vol. 37, No. 3, 2006, pp. 645-667.

SALOMÃO FILHO, Calixto. Direito concorrencial: as estruturas. São Paulo: Malheiros, 1998. 
Direito concorrencial: as condutas. São Paulo: Malheiros, 2007.

SALOP, Steven C. Exclusionary Conduct, Effect on Consumers and the Flawed ProfitSacrifice Standard. In: Antitrust Law Journal, 73, pp. 311-374, 2006.

Practices that (Credibly) Facilitate Oligopoly Co-ordination, New Developments in the Analysis of Market Structure, Ch. 9, Joseph E. Stiglitz \& G. Frank Mathewson eds., 1986.

SAMUELSON, Martha. PIANKOV, Nikita. ELLMAN, Brian. Assessing the Effects of Mostfavored Nation Clauses. In: ABA Section of Antitrust Law, 2012.

SARTO, Elisa Santos Coelho. Cartéis Hub-and-Spoke: coordenação horizontal or meio de restrições verticais. In: Revista de Defesa da Concorrência, Vol. 5, n. ${ }^{\circ}$ 2, novembro 2017.

SASS, Tim R. SAURMAN, David R. Mandated Exclusive Territories and Economic Efficiency: An Empirical Analysis of the Malt-Beverage Industry., vol. 36, In: Journal of Law and Economics, ${ }^{\circ} 153,1993$.

SCHUMPETER, Joseph A. Capitalismo, socialismo e democracia. Trad. Luiz Antônio Oliveira de Araújo. São Paulo: Editora da Unesp, 2017.

SCOTT MORTON, Fiona M. The strategic response by phamaceutical firms to the medicaid most-favoured nation customer rules. In: The RAND Journal of Economics, 28(2), pp. 269290. Disponível em: <https://www.jstor.org/stable/2555805>. Acesso em: 11/09/2018.

Contracts that Reference Rivals. In: Antitrust, Vol. 27, No. 3, 2013.

Disponível em: <http://www.crai.de/sites/default/files/publications/Contracts-that-reference-rivals.pdf $>$ Acesso em: 25/10/2018.

STENGER, S. Most-favoured-nation clauses and monopsonistic power: an unhealthy mix? In: American Journal of Law and Medicine, 15, pp. 111-128, 1989.

STUCKE, Maurice E. GRUNES, Allan P. Big Data and Competition Policy. In: Oxford University Press, 2016.

TIROLE, Jean. [Entrevista concedida a] David A. Price. In: Econ Focus, Fourth Quarter 2017. 
$<$ https://www.richmondfed.org/publications/research/econ_focus/2017/q4/interview> Acesso em: 27/09/2019.

VANDENBORRE, Ingrid. FRESE, Michel J. The Role of Market Transparency in Assessing MFN Clauses. In: World Competition, 38, No. 3, 2015, pp. 333-348.

VAN DOORN, Frederik. Resale Price Maintenance in EC Competition Law: the need for a standardised approach. University of Utrecht, School of Law, Department of European Law, Holanda, 2009. Disponível em: <https://papers.ssrn.com/sol3/papers.cfm?abstract_id=1501070>. Acesso em: 17/10/2018.

VEZZOSO, Simonetta. Online Platforms, Rate Parity and the Free Riding Defence. In: $11^{\text {th }}$ ASCOLA Conference, Leiden Law School, junho, 2016.

WALS, Francisca. SCHINKEL, Maarten Pieter. Platform Monopolization by Narrow-PPCBPG Combination: Booking et al. International Journal of Industrial Organization, 2018, p. 15. Disponível em:<https://ssrn.com/abstract=2985317> Acesso em: 25/09/2019.

WILLIAMSON, Oliver E. The mechanisms of governance. New York: Oxford University Press, 1996.

WRIGHT, Julian, One-Sided Logic in Two-Sided Markets. In: AEI-Brookings Joint Center Working Paper No. 03-10, setembro, 2003. Disponível em: <https://ssrn.com/abstract=459362>. Acesso em: 29/09/2018.

WANG, Chengsi. Search platforms: Showrooming and price parity clauses. 2016. Disponível em: <https://cepr.org/sites/default/files/Wright,\%20Julian\%20paper_0.pdf>. Acesso em: 17/10/2018.

WANG, Chengsi. Platform Investment and Price Parity Clauses. In: The Networks, Electronic Commerce and Telecommunications Institute, working paper \#16$17,2016$.

WU, Jason J. BIGELOW, John P. Competition and the Most Favored Nation Clause. CPI Antitrust Chronicle, 2013.

YURIEFF, Kaya. Instagram Stories is twice as popular as Snapchat, 28/06/2018. Disponível em: <https://money.cnn.com/2018/06/28/technology/instagram-stories-users/index.html> Acesso em: 03/11/2018. 
ZIMMER, Daniel and BLASCHCZOK, Martin, Most-Favoured-Customer Clauses and Two-Sided Platforms. In: Journal of European Competition Law \& Practice (JECLAP), Vol. 5, No. 4, 2014.

\section{OUTRAS REFERÊNCIAS}

ALEMANHA, Bundeskartellamt, 9th Decision Division B 9-121/13, p. 77. Disponível em: $<$ https://www.Bundeskartellamt.de/SharedDocs/Entscheidung/EN/Entscheidungen/Kartellv erbot/B9-121-13.pdf? _blob=publicationFile\&v=2> Acesso em: 08/08/2019.

Bundeskartellamt, $9^{\text {th }}$ Decision Division, B9-66/10, p. 17. Disponível em: $<$ https://www.Bundeskartellamt.de/SharedDocs/Entscheidung/EN/Entscheidungen/Kartellv erbot/B9-66-10.pdf\%3F_blob\%3DpublicationFile\%26v\%3D3> Acesso em: 07/08/2019.

Bundeskartellamt, Case ref. B6-46/12.

Bundeskartellamt. Vertical Restraints in the Internet Economy. Meeting of the Working Group on Competition Law, October, 2013. Disponível em: $<$ https://www.Bundeskartellamt.de/SharedDocs/Publikation/EN/Diskussions_Hintergrundp apiere/Vertical\%20Restraints\%20in\%20the\%20Internet\%20Economy.pdf? _ blob=publicat ionFile \&v=2> Acesso em: 10/09/2018.

Case report, 9 December 2013, Amazon removes price parity obligation for retailers on its Marketplace platform. Disponível em: $<$ http://www.Bundeskartellamt.de/SharedDocs/Entscheidung/EN/Fallberichte/Kartellverbot /2013/B6-46-12.pdf\%3F_blob\%3DpublicationFile\%26v\%3D2> Acesso em: 01/08/2019.

Monopolkomission. Competition Policy: The Challenge of Digital Markets. Special Report, No. 68, 2015. Disponível em: <http://www.monopolkommission.de/images/PDF/SG/s68_fulltext_eng.pdf $>$. Acesso em: 03/11/2018.

Monopolkomission. Special Report No 68: Competition policy: The challenge of digital markets - Special Report by the Monopolies Comission pursuante to section 44(1)(4) of the Act Against Restraints on Competition, 2015. Disponível em: $<$ http://www.monopolkommission.de/index.php/en/press-releases/52-competition-policythe-challenge-of-digital-markets $>$. Acesso em: 03/11/2018. 
APPLE. Apple Launches iPad. In: Apple, 27/01/2010. Disponível em: $<$ https://www.apple.com/newsroom/2010/01/27Apple-Launches-iPad/> Acesso em: 03/07/2019.

BRASIL. Acordo Geral de Tarifas e Comércio 1994. Disponível em: $<$ http://www.mdic.gov.br/comercio-exterior/negociacoes-internacionais/1885-omcacordos-da-omc>. Acesso em: 08/09/2018.

B2W. Termos e Condições Gerais do Marketplace. Disponível em: $<$ https://www.b2wmarketplace.com.br/docs/Termos-e-Condicoes-Gerais_MarketPlace_Modelo-A_01-06-17.pdf> Acesso em: 18/09/2019. B2W. Release de Resultados do 4 T2018. https://static.b2wdigital.com/upload/releasesderesultados/00003135.pdf> Acesso em: 18/09/2019.

Booking, Decolar e Expedia celebram acordo de cessação com o CADE. 27/03/2018. Em: <http://www.cade.gov.br/noticias/booking-decolar-e-expedia-celebramacordo-de-cessacao-com-o-cade> Acesso em: 21/09/2019.

CADE. Ato de Concentração n. $^{\circ}$ 08012.001234/2000-20. Requerentes: Ericsson Telecomunicações S/A e Emerson Electric do Brasil Ltda., Conselheiro Relator Márcio Felsky, j. 23/08/2000.

BRASIL. CADE. Ato de Concentração n. ${ }^{\circ}$ 08700.001390/2017-14, AT\&T Inc. e Time Warner Inc. Conselheiro Relator Gilvandro Vasconcelos Coelho de Araújo, j. 18/10/2017.

CADE. Ato de Concentração $n^{\circ}{ }^{0}$ 08700.006567/2015-07. Requerentes: Ball Corportion e Rexam PLC, Conselheiro Relator Gilvandro Vasconcelos Coelho de Araújo, j. $09 / 12 / 2015$.

CADE. Inquérito Administrativo n. ${ }^{\circ}$ 08012.009876/2007-79.

CADE. Procedimento Preparatório $n .^{\circ}$ 08700.005679/2016-13.

CADE. Processo Administrativo $n .^{\circ}$ 08012.002874/2004-14. Relator Conselheiro Alexandre Cordeiro de Macedo, j. 01/02/2017.

CADE. Processo Administrativo $n .^{\circ}$ 08012.004472/2000-12. Relator Conselheiro Olavo Zago Chinaglia, j. 01/10/2014. 
CADE. Processo Administrativo ${ }^{\circ}{ }^{\circ}$ 08012.006923/2002-18. Relator Conselheiro Ricardo Machado Ruiz, j. 20/02/2013.

CADE. Processo Administrativo . $^{\circ} 08012.007011 / 2006-97$. Relator Conselheiro Alexandre Cordeiro de Macedo, j. 19/04/2017.

CADE. Processo Administrativo $n .^{\circ}$ 08012.010932/2007-18. Relator Conselheiro Márcio de Oliveira Júnior, j. 25/02/2015.

CADE. Processo Administrativo 08012.000030/2011-50. Relator Conselheiro Alexandre Cordeiro de Macedo, j. 09/12/2015.

Carrefour. Termos e Condições Gerais do Marketplace. Disponível em: $<$ https://static.carrefour.com.br/imagens/banner-

html/bgs/2017/dezembro/mktplace/comissao-termos-e-

condicoes.pdf?crfimt=subhome\%7Cmarketplace\%7Cbn\%7Cbnfm\%7Cmarketplace termos -e-condicoes_carrefour_oferta_bnfm_mktplace_150319\%7C2\&cfrict=marketplace> Acesso em: 25/09/2019.

Cissa Magazine. Contrato de Comercialização de Produtos em Marketplace. Disponível em: <https://www.cissamagazine.com.br/contrato-cissa-marketplace.pdf $>$ Acesso em: 25/09/2019.

Colombo. Termos e Condições de Uso do Marketplace. Disponível em: $<$ https://images.colombo.com.br/hotsite/marketplacecolombo/pdf/termosmarketplacecolom bo2018.pdf> Acesso em: 25/09/2019.

Decorsale. Termos e Condições Gerais. Disponível em: < https://www.decorsale.com.br/termos-e-condicoes-gerais-de-marketplace> Acesso em: $25 / 09 / 2019$

Hotéis.com. Termos e Condições Gerais. Disponível em: < https://www.hoteis.com/customer_care/terms_conditions.html> Acesso em: 19/09/2019.

Itamaraty. Disponível em: <http://www.itamaraty.gov.br/pt-BR/politicaexterna/diplomacia-economica-comercial-e-financeira/132-organizacao-mundial-docomercio-omc $>$. Acesso em: 15/09/2018.

Martketbox. Termos e Condições de Uso do Marketplace. Disponível em: $<$ https://www.marketbox.com.br/termos-e-condicoes-de-uso-do-marketplace/> Acesso em: $25 / 09 / 2019$. 
Muzeez. Termos de Uso. Disponível em: <https://muzeez.com.br/termos-de-uso $>$ Acesso em: 25/09/2019.

Rock2You. Termos e condições de Uso. Disponível em: $<$ http://lojista.rock2you.com.br/pdf/rock2you-minuta-contrato-lojista.pdf> Acesso em: 25/09/2019.

Todamulher.com. Termos e Condições de Uso. Disponível em: < https://www.todamulher.com.br/termos-e-condicoes-de-uso-do-marketplace/> Acesso em: 25/09/2019.

COMISSÃO EUROPEIA. Antitrust: Commission announces the launch of market tests in investigations in the online hotel booking sector by the French, Swedish and Italian competition authorities, 15/12/2014. Disponível em: <https://europa.eu/rapid/pressrelease_IP-14-2661_en.htm> Acesso em: 15/08/2019.

Case 39847 E-Books. Disponível em: <http://ec.europa.eu/competition/elojade/isef/case_details.cfm?proc_code=1_39847> Acesso em: 23/07/2019. Caso COMP/38427 PO Pay Television Fil Output Agreements.

Disponível em:

$<$ <http://ec.europa.eu/competition/elojade/isef/case_details.cfm?proc_code=1_38427>

Acesso em: 10/09/2018.

European Comission, Case AT. 40153 E-books MFNs and related matters (Amazon). Disponível em: <http://ec.europa.eu/competition/antitrust/cases/dec_docs/40153/40153_4392_3.pdf> Acesso em: 01/08/2019.

Report on the monitoring exercise carried out in the online hotel bookng sector by EU competition authorities in 2016. 2017, Disponível em:

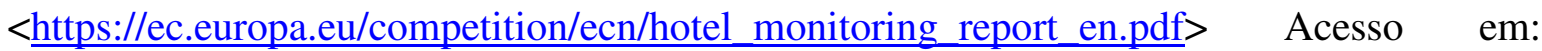
08/08/2018.

ESTADOS UNIDOS DA AMÉRICA. Apple v. United States, U.S. Supreme Court, No. 15565. 
Booking.com. Termos e Condições Gerais.

Disponível em: < https://www.booking.com/content/terms.pt-br.html> Acesso em: 19/09/2019.

Dr. Miles Medical Co. v. John D. Park \& Sons Co.,

220 U.S. 373 (1911).

Inc. v. Broadway-Hale Stores, Inc., 359 U.S. 207

(1959).

Leegin Creative Leather Products, Inc. v. PSKS Inc.,

551 U.S. 877 (2007).

Online Travel Co. Hotel Booking Antitrust Litig., 997

F. Supp. $2 d \quad 526 \quad$ (N.D. Tex. 2014). Disponível em:

$<$ https://law.justia.com/cases/federal/district-

courts/texas/txndce/3:2012cv03515/222550/136/> Acesso em: 05/09/2019.

Piazza v. Major League Baseball, 831 F. Supp. 420

(E.D. Pa. 1993).

Sherman Act. Disponível em:

< https://www.law.cornell.edu/uscode/text/15/1> Acesso em: 05/09/2019.

Standard Oil Co. of New Jersey v. United States, 221

U.S. 1 (1911).

Toys “R” Us, Inc. v. Federal Trade Commission, 221

F.3d 928 (2000).

United States v. Apple, Inc., 791 F.3d 290 (2d Cir.

2015).

United States v. Brown University, 5 F.3d 658, 669

(3d Cir. 1993);

United States v. General Electric Co., Case No.

28228, 1977, WL 1474.

FRANÇA. Autorité de la Concurrence: Press release 15 December 2014: Online hotel

Booking sector. dezembro de 2014. Disponível em: 
$<$ http://www.autoritedelaconcurrence.fr/user/standard.php?id_rub=592\&id_article=2463> Acesso em: 19/08/2019.

Eversheds Sutherland. France - Macron Law: A Focus on Online Hotel Reservation Platforms. 20/10/2015. Disponível em: https://www.evershedssutherland.com/global/en/what/articles/index.page?ArticleID=en/Competition_EU_and_Re gulatory/MACRON_LAW_FRANCE Acesso em: 14/08/2019.

ITÁLIA. Agenzie turistiche on line: avviata istruttoria nei confronti di Booking ed Expedia per verificare l'esistenza di violazioni concorrenziali. In: Autorità Garante della Concorrenza e del Mercato, 19/05/2014. Disponível em: <https://www.agcm.it/media/dettaglionotizia?id=77c78135-2ac9-43c9-bb46cf038d7b8eea\&parent=News\&parentUrl=/media/news > Acesso em: 14/08/2019.

Impegni di Booking all'Antitrust sui prezzi offerti dagli hotel partner. In: Autorità Garante della Concorrenza e del Mercato, 15/12/2014. Disponível em: $<$ https://www.agcm.it/media/dettaglio-notizia?id=75444112-24c4-47a5-900f714203cd4691\&parent=News\&parentUrl=/media/news $>$ Acesso em: 14/08/2019.

Prenotazioni on line impegni di Booking chiusa l'istruttoria in Italia, Francia e Svezia. In: Autorità Garante della Concorrenza e del Mercato, 21/04/2015. Disponível em: $<$ https://www.agcm.it/media/dettaglio-notizia?id=26552259-e74f-42c6-97d1-

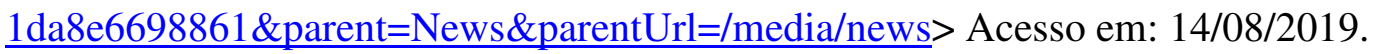

Legge 4 agosto 2017, n. 124 - Legge annual per il mercato e la concorrenza. Disponível em: https://www.normattiva.it/uri-res/N2Ls?urn:nir:stato:legge:2017;124 Acesso em: 20/08/2019.

UNIÃO EUROPEIA. Associação de Hotéis, Restaurantes e Cafés da Europa (HOTREC Hospitality Europe). European Hotel distribution study, 2017. Disponível em: $<$ https://www.hotrec.eu/wp-content/customerarea/storage/2a67daccb0e9486218e1a53b48494ab8/European-hotel-distribution-studyfinal-results-revsl18.pdf> Acesso em: 19/09/2019.

Corte Europeia. Case C-67/13 P Groupement des Cartes Bancaires v. Commission, ECLI:EU:C:2014:2204, § 53; Case C-286/13 P Dole v Commission, ECLI:EU:C:2015:184, § 117; Joined Cases C-501/06 P, C-513/06 P, C-515/06 P and C519/06 P GlaxoSmithKline, ECLI:EU:C:2008:738, § 58; Joined Cases 96/82 to 102/82, 
104/82, 105/82, 108/82 and 110/82 IAZ International Belgium and Others ECLI:EU:C:1983:310, § 25.

Tratamento sobre o Funcionamento da União Europeia (TFEU).

Disponível

em:

$<$ http://www.concorrencia.pt/vPT/A_AdC/legislacao/Documents/Europeia/Tratado_Funcio namento_U_E.pdf> Acesso em: 23/07/2019.

Vertical Restraints Block Exemption Regulation - Commission Regulation (EU) No 330/2010 of 20 April 2010 on the application of Article 101(3) of the Treaty on the Functioning of the European Union to categories of vertical agreements and concerted practices. Disponível em: <https://eur-lex.europa.eu/legalcontent/EN/ALL/?uri=CELEX\%3A32010R0330> Acesso em: 07/08/2019. 